\title{
Health care costs, work productivity and activity impairment in non-malignant chronic pain patients
}

\author{
Christian Kronborg • Gitte Handberg • \\ Flemming Axelsen
}

Received: 20 April 2007/Accepted: 10 January 2008/Published online: 7 February 2008

(C) The Author(s) 2008

\begin{abstract}
This study explores the costs of non-malignant chronic pain in patients awaiting treatment in a multidisciplinary pain clinic in a hospital setting. Health care costs due to chronic pain are particular high during the first year after pain onset, and remain high compared with health care costs before pain onset. The majority of chronic pain patients incur the costs of alternative treatments. Chronic pain causes production losses at work, as well as impairment of non-work activities.
\end{abstract}

Keywords Chronic pain - Costs - Work productivity · Activity impairment

\section{Introduction}

While the clinical effectiveness of multidisciplinary pain management in chronic non-malignant pain patients has been demonstrated in a number of studies [3, 9, 21], the cost-effectiveness of multidisciplinary pain management has not been determined, except in relation to multidisciplinary treatment in specific chronic pain patient groups $[16,20]$.

C. Kronborg ( $\square)$

Institute of Public Health, Health Economics Unit,

University of Southern Denmark, J.B. Winsløwsvej 9B, 5000 Odense C, Denmark

e-mail: cka@sam.sdu.dk

\section{G. Handberg}

Funen County Multidisciplinary Pain Clinic, Odense University

Hospital, Søndre Boulevard 29, 5000 Odense C, Denmark

F. Axelsen

Coloplast A/S, Global Marketing, Holtedam 1,

3050 Humlebaek, Denmark
Chronic pain management is typically facilitated in multidisciplinary pain clinics in a hospital setting. Based on heterogeneous pain pathophysiology and aetiology [2], treatment at pain clinics consists of different combinations of physical, psychological, educational, behavioural, and cognitive interventions, and is adapted to the individual patient. Generally, patients will have suffered from chronic pain for several years before being referred to a clinic. Often referral is not introduced before all other alternatives have been shown unsuccessful [20].

Decision makers may consider the cost-effectiveness of clinics, rather than the cost-effectiveness of treating specific patient groups, e.g. patients with chronic low back pain, when allocating resources. Whereas studies of the costeffectiveness of treatment targeted at specific patient groups can be designed as traditional randomised controlled trials, economic evaluation of multidisciplinary pain clinics involves several methodological challenges since controlled experiments may be difficult or even impossible to carry out.

The overall cost-effectiveness of an intervention can be established in two ways. Firstly, the cost of intervention is offset by reductions in other resource uses, leading to an overall decline in the cost of pain management, together with unaltered or improved patients health and well-being in general. Secondly, the intervention may cause an increase in costs together with an improvement in patient health and well-being. That is to say, the cost of the intervention is not offset by reductions in the use of other resources. In that case, decision makers must decide whether additional costs are acceptable in relation to improvement in patient health and well-being [6]. In the latter case, intervention cost-effectiveness is based on judgement.

Whether pain management in a multidisciplinary pain clinic can reduce health care costs and other costs related to 
chronic pain may depend on several factors, for example the extent to which the clinic can control or influence the use of health care resources, e.g. hospitalisation. The potential for cost savings as a result of multidisciplinary pain management may also depend on resource use at the onset of treatment in a pain clinic, i.e. the higher the health care costs at the outset, the higher the potential for cutting costs.

The aim of this study is to explore the costs of nonmalignant chronic pain in patients awaiting treatment in a multidisciplinary pain clinic in a hospital setting.

\section{Methods}

\section{Study design}

The study was designed as a cross sectional study of patients referred from general practice for treatment at a multidisciplinary pain hospital clinic. We sampled all patients on the waiting list for treatment at The Multidisciplinary Pain Clinic in Funen County at Odense University Hospital by 1 December 2005. The target was to include 200 patients in the study. Because it was not possible to reach this target by that date, we included patients referred to the clinic's waiting list until 18 January 2006. Data were collected by a telephone interview and mailed questionnaire. Inclusion of participants was terminated when it was confirmed that 200 interviews had been performed. The telephone interviews were structured by a questionnaire, and carried out by personnel from the Multidisciplinary Pain Clinic in Funen County at Odense University Hospital experienced in contact with this patient group. Patients that would have needed assistance from an interpreter during a telephone interview, i.e. patients that were not fluent in Danish, were excluded from the sample.

From the referral notes, we collected information on gender, date of birth and referral. The referral notes also provided details of pain location, pain diagnosis and aetiology. The data were merged with data from public registers, including data on participant use of in- and outpatient treatment and visits to hospital accident and emergency wards. Public register data contains information about all discharges of individual patients from publicly owned hospitals, including outpatient visits and accident and emergency department visits [1, 13]. All participant records were extracted from 1 January 1996 to 31 December 2005.

Each discharge was classified in accordance with the Danish Diagnosis Related Grouping (DRG) system [18]. Discharges before 2000, i.e. before the introduction of the Danish DRG system, were classified according to the system that applied for 2000. For discharges in 2001 and later, the current DRG system was applied [19].

Data on the use of services delivered by general practitioners and other medical specialists, dentists, physiotherapists, chiropractors, chiropodists, and psychological counsellors was obtained from The National Health Insurance Service Registry, which is a central register of health care services covered by Danish national health insurance [14]. The register contains information on service type, cost and delivery date. All records covering the same period as for hospital services were extracted from the register.

Data on participants use of prescription drugs was collected from a Danish prescription register, The Odense University Pharmaco-epidemiological Database (OPED), which is a register based on transaction reports from the dispensing pharmacies in Funen County [10, 11]. The transaction reports were identifiable from participant personal identification numbers. From each transaction report, we extracted information on the date the prescription was presented at the pharmacy, and the number, type and cost of the medication(s) prescribed. We extracted data covering 1 January 1996 to 31 December 2005. Since OPED covers the County of Funen, only data on participants living in that county during this period was retrieved. Data on over-the-counter medication was not available from OPED.

Data on participant use of public sector health care services was collected during the telephone interview. Participants were asked whether they received assistance with regard to personal care/hygiene and/or medication administration due to their chronic pain diagnosis. They were also asked whether they received assistance with house cleaning, laundry or shopping (services that are typically provided free of charge for chronically impaired persons in Denmark). Participants reported the year in which they began receiving these types of services. Finally, participants were asked whether they had chosen to pay for private house cleaning, gardening, and/or other services as a result of chronic pain. Participants that had paid for private services were asked to disclose their total expenditures for the 6 month period prior to the interview.

In a mailed questionnaire, participants were asked whether they had used alternative treatments, such as homeopathy, reflexology, acupuncture, healing or hypnosis. Where relevant, participants were asked to disclose their spending on alternative treatments.

\section{Costs}

The resource volumes were combined with unit costs to obtain a cost per person. The unit costs were included as an 
approximation of the social opportunity cost of each item [5]. All costs were measured in 2006 values of Danish kroner (DKK). Where required, costs were converted to 2006 values using the official consumer price index published by Statistics Denmark [17].

We used DRG charges as unit costs for hospital treatment. DRG charges were provided by the Danish National Board of Health. DRG charges reflect the average hospital costs for treating patients with similar conditions and processes of care. For patients staying beyond the number of days covered by the DRG charge, an extra charge per day for the excess number of days covered was added to the hospital costs.

The unit costs of GP services and services from other healthcare providers were based on the prevailing national health insurance rate (data included the fee reimbursed at the point of delivery and type of resource used). We added patient co-payment for physiotherapy, chiropractic treatment, and dental services as appropriate.

The prevailing package price for prescription drugs was provided along with the data on prescription drug consumption. The package price reflected the retail price, which included the cost price for the pharmacy, the prescription fee charged by the pharmacy and value added tax before any reimbursement at the point of delivery. Thus, the reported price covered the full product cost irrespective of who incurred the cost.

Unit costs relating to personal care and practical assistance in the home were collected from a national database that collects information from every Danish municipality regarding costs per hour for these services. Since they are provided free of charge for the recipient, we assumed that the unit cost of these services reflected the opportunity cost. We used the unit cost applicable to the council where the participant lived. As unit costs vary between municipality, we analysed how different unit costs affected the results, using the minimum and maximum observed unit costs of 213 and 334 DKK per hour, for sensitivity analysis.

In order to report annual costs, we multiplied the reported resource use of personal and practical assistance in the home by 12 .

The costs of privately provided house cleaning, gardening, and/or other services, as well as the costs of alternative treatment were estimated on the basis of participant expenditure on these services. That is to say, we assumed that the full cost of these services and alternative treatments was met by participants.

\section{Productivity costs}

Productivity costs were defined as costs due to lost or impaired ability to work or to engage in leisure activities due to chronic pain [12], i.e. the costs of lost production, paid as well as unpaid.

We included a specific health problem version of the work productivity and activity impairment (WPAI) instrument in the postal questionnaire [15]. The WPAI instrument was used to determine the extent of time lost from work and productivity loss whilst at work due to chronic pain in participants in the work force. It was also used to determine the extent to which participants were able to carry out regular daily activities, other than work at a job such as housekeeping, or child caring (see Appendix).

All questions in the WPAI instrument related to the preceding 7 days. The questions considered the number of hours missed from work due to chronic pain, the number of hours missed because of other reasons (such as time off or holidays), hours actually worked and productivity while working.

Productivity was measured using a scale ranging from zero (chronic pain had no effect on work) to ten (chronic pain completely prevented working). A similar rating scale was used to assess participant ability to carry out regular daily activities other than paid work.

For respondents that were working, we estimated the percentage of work time missed due to chronic pain, the percentage of impairment while working due to chronic pain, and the percentage of overall work impairment due to chronic pain. Work time was defined as the number of hours participants actually worked or should have worked if they had not missed work due to chronic pain. That is to say, work time net of vacation, holidays and time off.

Furthermore, we estimated the percentage of activity impairment due to chronic pain for all participants.

\section{Analysis}

We used a panel data analytic approach to analyse the effect of pain duration on health care prescription drug use. That is to say, we calculated participant individual annual health care and prescription drug use costs from 1995 to 2005. This data was combined with information relating to how many years the participant had suffered from chronic pain. Thus, each participant produced ten observations.

For participants that had suffered from pain for exactly 10 years, the first observation (i.e. 1995) would be the first year with pain, the second observation the second year and so on. For participants that had suffered from pain for less than 10 years, some observations were for years before pain onset. For patients that had suffered from chronic pain for more than 10 years, pain onset was before 1995 .

Thus, the ten observations per participant covered a time span from 0 to 9 years with chronic pain, over a time span from 40 to 50 years with chronic pain, and down to -9 to 
1 year with chronic pain (negative values indicating time in which the participant did not suffer from chronic pain).

We used pooled ordinary least squares (OLS) regression, and fixed and random effects models to explore how pain duration affected annual health care and prescription drug use costs.

The pooled OLS model was estimated using ordinary least squares on the cross-sectional time-series data for all individuals. It was assumed that all individual-specific effects were constant and equal across the participants, i.e. it assumed heterogeneity across participants. Thus, only a single overall intercept was estimated. This assumption was relaxed using the fixed effect model, which captured individual specific effects that do not change over time.

In the random effects model, the individual specific effects were treated as randomly distributed. However, it was assumed that the individual effects were uncorrelated with other independent variables in the model.

Participant age and gender were used as independent variables. Pain duration was included as a categorical variable to capture non-linear effects on health care and prescription drug costs. That is to say, pain duration was included as dummy variables, with a value of 0 or 1 . A variable that equalled 1 was used if the observation considered related to the year before pain onset (otherwise the variable equalled 0 ), another variable equal to 1 was used if the participant had suffered from pain over a period of up to 1 year, and a third variable equal to 1 if the participant had suffered from pain between 1 and 2 years, and so forth. However, pain duration above 5 years was categorised in 5 -year intervals. For example, a variable that equalled 1 was used if the participant had suffered from chronic pain for between 5 and 10 years.

The dummy variables measured the differences in health care costs and prescription drug costs over a particular time and reference period, namely the $1-9$ years before pain onset.

We successively tested whether the pain duration coefficients relating to the dummy variables differed and, as the coefficients for the remaining dummies were not statistically different from each other, ended up including only three dummy variables for pain duration in the regression analyses of health care costs and only two in the regression analyses of prescription drug costs.

The variables for age, gender, and pain duration constituted the main effects of the model. Because of the dummy variables, coefficients referred to a reference person, namely a male with 1 year or more since pain onset.

Furthermore, we included year dummies for 1996 to 2005 in order to capture the fact that the population may have different distributions in different time periods [23]. However, the first dummy variable for year 1996 was left out, i.e. the year 1996 was used as a reference.
A Hausmann test was used to compare random and fixed effects estimators [23].

\section{Results}

Participant characteristics

A total of 298 patients were on the waiting list for treatment at The Multidisciplinary Pain Clinic in Funen County at Odense University Hospital. Of these, 94 patients were not included in the study: 14 because the sample size target was met, 22 who did not give consent, 21 were not at home when contacted, 5 due to non-fluency in Danish, 11 were not listed in the telephone lists, 6 were withdrawn from the waiting list, and 15 were not included due to other reasons. This left 204 participants for the study.

Table 1 shows participant pain characteristics. The majority of participants were females $(61 \%)$. At the time of the telephone interview, they were between 20 and 89 years of age, with a mean age of 48.1 years $(S D=13.74)$. The enrolled patients did not differ on age or gender from those patients that were not included in the study.

Table 1 Pain characteristics of the participants. Figures represent number of persons with percentages in parenthesis $(n=204)$

\begin{tabular}{|c|c|c|}
\hline \multicolumn{3}{|l|}{ Pain location } \\
\hline Head, neck, shoulders, arms & 114 & $(56 \%)$ \\
\hline Thorax & 10 & $(5 \%)$ \\
\hline Abdomen & 18 & $(9 \%)$ \\
\hline Lower back and legs & 122 & $(60 \%)$ \\
\hline \multicolumn{3}{|l|}{ Pain diagnosis } \\
\hline Neuropathic & 56 & $(28 \%)$ \\
\hline Vescical & 24 & $(12 \%)$ \\
\hline Muscles, bones, joint, connective tissue & 163 & $(80 \%)$ \\
\hline Skin (nociceptive pain) & 2 & $(1 \%)$ \\
\hline Unknown & 6 & $(3 \%)$ \\
\hline \multicolumn{3}{|l|}{ Aetiology } \\
\hline Congenital & 5 & $(3 \%)$ \\
\hline Trauma, operation, burn & 67 & $(33 \%)$ \\
\hline Degeneration & 81 & $(40 \%)$ \\
\hline Infection, inflammation & 9 & $(4 \%)$ \\
\hline Other/inexplicable & 79 & $(39 \%)$ \\
\hline \multicolumn{3}{|l|}{ Pain duration (years) } \\
\hline $0-4$ & 65 & $(32 \%)$ \\
\hline $5-9$ & 50 & $(25 \%)$ \\
\hline $10-14$ & 25 & $(12 \%)$ \\
\hline $15-19$ & 18 & $(9 \%)$ \\
\hline $20-24$ & 20 & $(10 \%)$ \\
\hline $25-29$ & 11 & $(5 \%)$ \\
\hline $30-39$ & 10 & $(5 \%)$ \\
\hline$>40$ & 5 & $(3 \%)$ \\
\hline
\end{tabular}


Participants reported that they had suffered from chronic pain from between 6 months and up to 50 years with an average of 11.4 years ( $\mathrm{SD}=10.06$; median 7.5 years). Female participants had suffered from chronic pain for 3.6 years more than male participants $(95 \%$ confidence interval: 0.9-6.2).

Consequently, female participants were younger than male participants at pain onset: the female participants were on average 34.7 years old $(\mathrm{SD}=14.77)$, whereas male participants were 40.5 years old $(\mathrm{SD}=15.16$; $P=0.0045)$.

The most frequent pain location was in the lower back, head, neck, shoulders or arms. The most frequent pain diagnoses were nociceptive pain in muscles, bones, joints or connective tissue. Pain was mainly caused by degeneration, and trauma, operation, or burns. However, pain aetiology in many participants was unknown.

Health care costs

Table 2 shows the results from the regression analyses of annual health care costs in relation to age, gender, and pain duration.

The Hausman specification test found no statistically significant difference between the random effects and fixed effects estimates, and thus no conclusive evidence as to whether the fixed or random effects estimates were preferred.

Whereas gender did not affect annual health care costs, annual health care costs increased with age by about DKK 560 per person per year according to the pooled OLS and random effects models, and by about DKK 800 per person per year according to the fixed effects model.

The annual health care costs were higher in years after pain onset than before pain onset. Furthermore, health care costs were higher in the year before reported pain onset than in previous years, i.e. 9 to 2 years before pain onset. The pooled OLS model suggested that the annual health care costs in the year prior to pain onset were DKK 8,699 per person higher than in previous years. However, at a $5 \%$ level of significance in the fixed and random effects models the coefficient to this variable was not significant.

In the year in which participants reported pain onset, the annual health care costs were about DKK 17,500 per person higher than health care cost in the period 2 to 9 years prior to pain onset. The exact figure depended on the regression model.

In years where the participants had suffered from chronic pain for more than 1 year, the annual health care costs were about DKK 8,000 per person higher than in years prior to pain onset. However, at a 5\% level of

Table 2 Regression of annual health care costs on gender, age, and pain duration (number of observations $=2,040$; number of groups $=204$ )

\begin{tabular}{|c|c|c|c|c|c|c|c|c|c|}
\hline & \multicolumn{3}{|l|}{ Pooled OLS } & \multicolumn{3}{|c|}{ Random effects (RE) } & \multicolumn{3}{|c|}{ Fixed effects (FE) } \\
\hline & Coefficient & SE & $P$ & Coefficient & SE & $P$ & Coefficient & SE & $P$ \\
\hline Female & 411 & 2,151 & 0.849 & 465 & 3,932 & 0.906 & - & - & - \\
\hline Age & 560 & 91 & 0.000 & 562 & 140 & 0.000 & 806 & 526 & 0.125 \\
\hline \multicolumn{10}{|l|}{ Duration } \\
\hline-1 to 0 years & 8,699 & 4,013 & 0.030 & 8,456 & 4,451 & 0.057 & 8,290 & 4,507 & 0.066 \\
\hline 0 to 1 year & 18,421 & 5,702 & 0.001 & 17,519 & 4,315 & 0.000 & 17,117 & 4,426 & 0.000 \\
\hline 1 year or more & 8,715 & 1,846 & 0.000 & 8,094 & 3,434 & 0.018 & 7,597 & 4,099 & 0.064 \\
\hline d97 & -1678 & 3,468 & 0.629 & -1682 & 4,093 & 0.681 & $-1,908$ & 3,881 & 0.623 \\
\hline d98 & -2572 & 3,691 & 0.486 & -2435 & 4,096 & 0.536 & $-2,984$ & 3,714 & 0.422 \\
\hline d99 & -1911 & 3,611 & 0.597 & -1845 & 4,140 & 0.656 & $-2,511$ & 3,614 & 0.487 \\
\hline $\mathrm{d} 00$ & -3391 & 4,050 & 0.403 & -3268 & 4,176 & 0.434 & $-4,144$ & 3,555 & 0.244 \\
\hline $\mathrm{d} 01$ & 1,877 & 3,759 & 0.618 & 2,041 & 4,268 & 0.632 & 962 & 3,564 & 0.787 \\
\hline $\mathrm{d} 02$ & 1,937 & 4,157 & 0.641 & 2,176 & 4,376 & 0.619 & 900 & 3,632 & 0.804 \\
\hline $\mathrm{d} 03$ & 16,543 & 5,950 & 0.005 & 16,842 & 4,491 & 0.000 & 15,370 & 3,735 & 0.000 \\
\hline d04 & 16,245 & 5,885 & 0.006 & 16,569 & 4,588 & 0.000 & 14,886 & 3,878 & 0.000 \\
\hline d05 & 1,598 & 4,389 & 0.716 & 1,914 & 4,670 & 0.682 & - & - & - \\
\hline Constant & $-9,032$ & 4,141 & 0.029 & $-8,838$ & 7,008 & 0.207 & $-17,778$ & 21,490 & 0.408 \\
\hline$R^{2}$ & 0.0729 & & & 0.0729 & & & 0.0704 & & \\
\hline Root MSE & 47,476 & & & & & & & & \\
\hline \multicolumn{10}{|l|}{ Specification test } \\
\hline \multicolumn{3}{|c|}{ Hausman test for RE versus FE } & \multicolumn{7}{|c|}{$X^{2}(12)=0.69 ; P=1.000$} \\
\hline
\end{tabular}

$O L S$ ordinary least squares 
significance the coefficient was not statistically significant in the fixed effects model.

\section{Prescription drugs}

Table 3 shows the results of regression analyses of annual prescription drug costs in relation to gender, age, and pain duration. Gender did not influence the costs, whereas annual prescription drug costs increased with age. However, at a 5\% level of significance, the coefficients to the variables for pain duration were not statistically significant, except for the coefficient for pain duration for more than 1 year, in the pooled OLS model, which suggested that prescription drugs costs were DKK 2,466 per person higher compared with drug consumption costs in years prior to pain onset.

\section{Council services}

A total of nine participants $(4 \%)$ reported that they received council personal services (e.g. personal care, dressing, medication). Furthermore, 26 participants (13\%) reported that they received council housekeeping and gardening services.
Use of personal care services and housekeeping and gardening services was decidedly skewed. Recipient use of personal care varied between 2 and $450 \mathrm{~h}$ per month. Leaving out the participant in receipt of $450 \mathrm{~h}$ personal care per month, the average amount of personal care was $35.4 \mathrm{~h}$ per recipient per month $(\mathrm{SD}=33.02)$.

Recipient use of housekeeping and gardening services ranged from between 1 and $56 \mathrm{~h}$ per month, averaging $6.8 \mathrm{~h}$ per recipient per month $(\mathrm{SD}=11.20)$.

Recipients had received council services and support for between 0 and 13 years at the time of the interview, with an average of 3.2 years per recipient $(\mathrm{SD}=3.58)$. Recipients had started receiving council services and support between 1 and 48 years after pain onset. On average, they started receiving help after 14.2 years after pain onset $(\mathrm{SD}=13.13)$.

Table 4 shows the average cost of council services with a sensitivity analysis since unit costs of services vary between municipalities in Denmark.

\section{Privately provided services}

Seventy eight $(38 \%)$ of the participants reported that they had paid for private housekeeping services and gardening.

Two participants reported that they had procured these services before pain onset. The remaining participants had

Table 3 Regression of annual costs of prescription drug use on gender, age, and pain duration (number of observations = 1,740; number of groups $=174)$

\begin{tabular}{|c|c|c|c|c|c|c|c|c|c|}
\hline & \multicolumn{3}{|l|}{ Pooled OLS } & \multicolumn{3}{|c|}{ Random effects (RE) } & \multicolumn{3}{|c|}{ Fixed effects (FE) } \\
\hline & Coefficient & $\mathrm{SE}$ & $P$ & Coefficient & SE & $P$ & Coefficient & SE & $P$ \\
\hline Female & 116 & 578 & 0.841 & 361 & 1,236 & 0.770 & - & - & - \\
\hline Age & 269 & 29 & 0.000 & 277 & 43 & 0.000 & 1,144 & 120 & 0.000 \\
\hline \multicolumn{10}{|l|}{ Pain duration } \\
\hline 0 to 1 year & 42 & 638 & 0.948 & -768 & 993 & 0.440 & $-1,116$ & 1,002 & 0.266 \\
\hline 1 year or more & 2,466 & 463 & 0.000 & 131 & 794 & 0.869 & -839 & 879 & 0.340 \\
\hline d97 & -295 & 638 & 0.644 & -181 & 959 & 0.850 & -998 & 907 & 0.272 \\
\hline $\mathrm{d} 98$ & -106 & 693 & 0.879 & 58 & 961 & 0.952 & $-1,600$ & 869 & 0.066 \\
\hline d99 & -501 & 688 & 0.466 & -235 & 968 & 0.808 & $-2,715$ & 844 & 0.001 \\
\hline $\mathrm{d} 00$ & -442 & 740 & 0.550 & -60 & 980 & 0.951 & $-3,353$ & 831 & 0.000 \\
\hline $\mathrm{d} 01$ & 143 & 799 & 0.858 & 699 & 998 & 0.484 & $-3,387$ & 830 & 0.000 \\
\hline $\mathrm{d} 02$ & 458 & 822 & 0.577 & 1,206 & 1,024 & 0.239 & $-3,663$ & 844 & 0.000 \\
\hline $\mathrm{d} 03$ & 407 & 837 & 0.626 & 1,385 & 1,057 & 0.190 & $-4,252$ & 869 & 0.000 \\
\hline $\mathrm{d} 04$ & 701 & 871 & 0.421 & 1,864 & 1,091 & 0.087 & $-4,559$ & 907 & 0.000 \\
\hline $\mathrm{d} 05$ & 5,956 & 2,086 & 0.004 & 7,238 & 1,119 & 0.000 & - & - & - \\
\hline Constant & -8455 & 1,315 & 0.000 & $-7,992$ & 2,128 & 0.000 & $-41,181$ & 4,990 & 0.000 \\
\hline$R^{2}$ & 0.1510 & & & 0.1452 & & & 0.1146 & & \\
\hline Root MSE & 11,578 & & & & & & & & \\
\hline \multicolumn{10}{|l|}{ Specification test } \\
\hline \multicolumn{3}{|c|}{ Hausman test for RE verses FE } & \multicolumn{7}{|c|}{$X^{2}(11)=6.67 ; P=0.8251$} \\
\hline
\end{tabular}


Table 4 Mean cost of council personal care, housekeeping and gardening services in Danish kroner (DKK) per participant per year. $n=204$ (figures in parentheses represent standard deviation)

\begin{tabular}{|c|c|c|c|}
\hline \multirow[t]{2}{*}{ Service } & \multirow{2}{*}{$\begin{array}{l}\text { Applying council } \\
\text { specific unit costs }\end{array}$} & \multicolumn{2}{|l|}{ Sensitivity analysis ${ }^{\mathrm{a}}$} \\
\hline & & $\begin{array}{l}\text { Applying lowest } \\
\text { observed unit cost }\end{array}$ & $\begin{array}{l}\text { Applying highest } \\
\text { observed unit costs }\end{array}$ \\
\hline Personal care & $12,468(110,172)$ & $9,444(86,028)$ & $14,964(136,296)$ \\
\hline Housekeeping, gardening, etc. & $2,592(144,544)$ & $2,136(11,376)$ & $3,348(17,844)$ \\
\hline Total & $15,060(111,312)$ & $11,568(86,916)$ & $18,300(137,688)$ \\
\hline
\end{tabular}

a The sensitivity analysis shows how the estimated cost changed when using unit costs applicable to different Danish councils

suffered from pain for an average of 7.7 years before they started procuring privately provided services $(\mathrm{SD}=9.76)$. At the time of the telephone interview, they had procured these services for an average of $4.5(\mathrm{SD}=5.75)$ years per person.

Forty one (20\%) had paid for such services within 6 months of the telephone interview. Reported expenditure was between DKK 90 and DKK 75,000. The average annual cost for privately provided services due to chronic pain was estimated at DKK 12,408 per participant $(\mathrm{SD}=67,356)$.

\section{Alternative treatment}

Out of 166 participants that returned the mailed questionnaire, $131(79 \%)$ reported that they had been treated by therapists outside the general health service (alternative treatment). Table 5 shows the various types of therapists contacted by participants as a result of chronic pain.

Eight (4\%) participants on the mailed questionnaire had contact with one or more alternative therapists before pain onset. Those that contacted alternative therapists after pain onset initiated alternative treatment between 0 and 40 years after pain onset. The average number of years between pain

Table 5 Use of treatments outside the general health service system (alternative treatments) at any time due to chronic pain. The figures represent the number of persons who have used the service (figures in parentheses represent percentages). $n=166$

\begin{tabular}{ll}
\hline Natural medicinal products (e.g. homeopathy) & $36(22 \%)$ \\
Reflexology & $51(31 \%)$ \\
Relaxation & $37(22 \%)$ \\
Instruction regarding diet, exercise etc. & $34(24 \%)$ \\
Acupuncture & $71(43 \%)$ \\
Touching & $9(5 \%)$ \\
Massage/manipulation & $70(42 \%)$ \\
Use of apparatus (e.g. magnetic passes) & $8(5 \%)$ \\
Healing & $39(24 \%)$ \\
Hypnosis & $6(4 \%)$ \\
Other & $52(31 \%)$ \\
\hline
\end{tabular}

onset and contact to alternative therapists was 6.3 years per person $(\mathrm{SD}=7.92)$.

Annual expenditure by users on alternative treatment was between DKK 300 and DKK 30,300. Thus, average expenditure on alternative treatment was DKK 2,978 per participant per year $(\mathrm{SD}=5,347)$.

\section{Production costs}

A total of 47 (29\%) participants reported they were currently employed. Table 6 shows the WPAI instrument results. On average, chronic pain meant that participants in work missed $19.4 \%$ of the time they could have worked $(\mathrm{SD}=32.93)$. During the time they actually worked, productivity was reduced by an average of 51.1 ( $\mathrm{SD}=$ 23.49), i.e. $31 \mathrm{~min}$ of every hour were not productive due to chronic pain. Out of the total number of work hours available to the respondent, $41.0 \%$ were lost due to chronic pain $(\mathrm{SD}=23.00)$.

All participants were asked about their ability to carry out non-work activities such as housekeeping, childcare, and studying. On average, they were impaired by $71.0 \%$ $(\mathrm{SD}=20.74)$, i.e. for every hour available for non-work activities, 42 min were lost due to chronic pain.

\section{Discussion}

This study shows that health care costs due to chronic pain are particular high during the first year after pain onset, and that the health care costs remain high compared with chronic pain patients' health care cost before pain onset. There is no conclusive evidence that chronic pain increases the costs of prescription drugs.

This study also shows that chronic pain causes production losses and impairment in non-work activities such as housekeeping, childcare, and studying.

An important strength of this study is that it relies on data from public registers relating to the use of health care services and prescription drugs. These types of registers 
Table 6 Work productivity and activity impairment during the previous 7 days

\begin{tabular}{|c|c|c|c|c|}
\hline & Number of observations & \multicolumn{2}{|c|}{ Mean (SD) } & [Min-Max] \\
\hline \multicolumn{5}{|l|}{ Patients in work $(n=47)$} \\
\hline Percent work time missed due to chronic pain & 43 & 19.4 & $(32.93)$ & {$[0-100]$} \\
\hline Percent impairment while working due to chronic pain & 43 & 51.1 & $(23.49)$ & {$[0-100]$} \\
\hline Percent overall work impairment due to chronic pain & 41 & 41.0 & $(23.00)$ & {$[0-80]$} \\
\hline \multicolumn{5}{|l|}{ All participants $(n=166)$} \\
\hline Percent activity impairment due to chronic pain & 153 & 71.0 & (20.74) & [0-100] \\
\hline
\end{tabular}

generally have a high degree of completeness and validity $[1,10,11,14]$. However, register data considers treatment practice at the time the service was provided to the patient, which may differ from contemporary treatment practice, i.e. treatment provided in 1996 may differ from treatment provided in 2006. Furthermore, technology and productivity may change over time. Thus, cost estimates due to chronic pain in this study may be affected by these factors. Caution should be exercised when using these estimates in further analyses on the influence of interventions to prevent or treat chronic pain. Furthermore, over-the-counter drugs, and some prescription drugs not subject to reimbursement, are not recorded in the prescription register.

Another limitation of the study is that it relies on information that may be affected by recall bias. Patients may have had difficulty in specifying the exact time of pain onset. Recall bias may have caused the finding that the health care costs increased the year before pain onset. In addition, recall bias may also have influenced the cost estimates relating to publicly and privately provided nonhealth care services and alternative treatments.

The results of regression analyses show that only a minor part of the variation in health care costs and prescription drug costs could be explained by gender, age, and pain duration. A limitation of the study is that several factors that might explain additional variation are not observable, for example information about diagnostics, during the same period as that covered by the data.

The results of the regression analyses of health care costs showed very high cost increases in 2003 and 2004. We have explored the data for potential extreme observations. However, we did not find any such observations that may have affected the results.

Whereas the analyses showed consistent results on the effects of pain duration on health care costs, the consequences of pain duration on prescription drug costs changed in the various regression models. The statistically significant effect of pain duration of 1 year or more on prescription drug costs in the pooled OLS model may be caused by individual effects. This individual effect is accounted for in the random effects and fixed effects models.
Several cost-of-illness studies of pain have been published, e.g. [4, 7, 8, 22]. Compared with previous studies, we consider the cost of chronic pain in general, not pain due to specific pathophysiology or aetiology. Furthermore, this study differs from cost-of-illness studies in their traditional form, in that we have not sought to estimate the total cost of chronic pain in the total Danish patient population.

The results of this study cannot be used in a priority setting, because assessments of the health benefits of the resources allocated to treatment of patients have not been included. However, the cost estimates may be useful in an economic evaluation that considers the cost-effectiveness of treating chronic pain patients in a multidisciplinary pain clinic. Based on the results of this study, evidence of costeffectiveness of chronic pain interventions would be expected if an intervention is capable of reducing health care consumption rather than prescription drug use.

Acknowledgement Data collection for the study was financed by Pfizer Denmark ApS. Flemming Axelsen was outcomes research manager at Pfizer Denmark Aps at the time of conducting this study.

Open Access This article is distributed under the terms of the Creative Commons Attribution Noncommercial License which permits any noncommercial use, distribution, and reproduction in any medium, provided the original author(s) and source are credited.

\section{Appendix}

More information about work productivity and activity impairment (WPAI) instruments is available from (April 2007):

http://www.reillyassociates.net/Index.html.

\section{References}

1. Andersen, T.F., Madsen, M., Jorgensen, J., Mellemkjoer, L., Olsen, J.H.: The Danish national hospital register. A valuable source of data for modern health sciences. Dan. Med. Bull. 46 263-268 (1999)

2. Becker, N., Bondegaard, T.A., Olsen, A.K., Sjogren, P., Bech, P., Eriksen, J.: Pain epidemiology and health related quality of life in 
chronic non-malignant pain patients referred to a Danish multidisciplinary pain center. Pain 73, 393-400 (1997)

3. Becker, N., Sjogren, P., Bech, P., Olsen, A.K., Eriksen, J.: Treatment outcome of chronic non-malignant pain patients managed in a Danish multidisciplinary pain centre compared to general practice: a randomised controlled trial. Pain 84, 203-211 (2000)

4. Borghouts, J.A., Koes, B.W., Vondeling, H., Bouter, L.M.: Costof-illness of neck pain in The Netherlands in 1996. Pain 80, 629636 (1999)

5. Brouwer W.B., Rutten F., Koopmanschap M.: Costing in economic evaluations. In: Drummond M., McGuire A. (eds.) Economic Evaluation in Health Care. Merging Theory with Practice. Oxford University Press, Oxford (2001)

6. Drummond, M.F., Sculpher, M.J., Torrance, G.W., O’Brien, B., Stoddart, G.L.: Methods for the Economic Evaluation of Health Care programmes. Oxford University Press, Oxford (2005)

7. Ekman, M., Johnell, O., Lidgren, L.: The economic cost of low back pain in Sweden in 2001. Acta. Orthop. 76, 275-284 (2005)

8. Ekman, M., Jonhagen, S., Hunsche, E., Jonsson, L.: Burden of illness of chronic low back pain in Sweden: a cross-sectional, retrospective study in primary care setting. Spine 30, 1777-1785 (2005)

9. Flor, H., Fydrich, T., Turk, D.C.: Efficacy of multidisciplinary pain treatment centers: a meta-analytic review. Pain 49, 221-230 (1992)

10. Gaist, D., Sorensen, H.T., Hallas, J.: The Danish prescription registries. Dan. Med. Bull. 44, 445-8 (1997)

11. Hallas, J.: Conducting pharmacoepidemiologic research in Denmark. Pharmacoepidemiol. Drug Saf. 10, 619-623 (2001)

12. Luce, B., Manning, W., Siegel, J., Lipscomb, J.: Estimating costs in cost-effectiveness analysis. In: Gold, M.R., Siegel, J.E., Russel, L.B., Weinstein, M.C. (eds.) Cost-effectiveness in Health and Medicine, pp. 176-213. Oxford University Press, Oxford (1996)

13. Mosbech, J., Jorgensen, J., Madsen, M., Rostgaard, K., Thornberg, K., Poulsen, T.D.: Landspatientregisteret. Evaluering af datakvaliteten. [The national patient registry. Evaluation of data quality. In Danish.]. Ugeskr. Laeger. 157, 3741-3745 (1995)

14. Olivarius, N.F., Hollnagel, H., Krasnik, A., Pedersen, P.A., Thorsen, H.: The Danish national health service register. A tool for primary health care research. Dan. Med. Bull. 44, 449-453 (1997)

15. Reilly, M.C., Zbrozek, A.S., Dukes, E.M.: The validity and reproducibility of a work productivity and activity impairment instrument. Pharmacoeconomics 4, 353-365 (1993)

16. Skouen, J.S., Grasdal, A.L., Haldorsen, E.M., Ursin, H.: Relative cost-effectiveness of extensive and light multidisciplinary treatment programs versus treatment as usual for patients with chronic low back pain on long-term sick leave: randomized controlled study. Spine 27, 901-909 (2002)

17. Statistics Denmark. Consumer price index. Statistics Denmark, Statistikbanken. Available from: URL: http://www.statistik banken.dk

18. Sundhedsministeriet (Ministry of Health): Takstsystem 2002vejledning (Hospital charges 2002. In Danish). National Board of Health, Copenhagen (2001)

19. Sundhedsstyrelsen (National Board of Health): Takstsystem 2005-Vejledning (Hospital charges 2005. In Danish). National Board of Health, Copenhagen (2005)

20. Thomsen, A.B., Sorensen, J., Sjogren, P., Eriksen, J.: Economic evaluation of multidisciplinary pain management in chronic pain patients: a qualitative systematic review. J. Pain Symptom Manage. 22, 688-698 (2001)

21. Thomsen, A.B., Sorensen, J., Sjogren, P., Eriksen, J.: Chronic non-malignant pain patients and health economic consequences. Eur. J. Pain 6, 341-352 (2002)

22. van Tulder, M.W., Koes, B.W., Bouter, L.M.: A cost-of-illness study of back pain in The Netherlands. Pain 62, 233-240 (1995)

23. Wooldridge J.M.: Econometric Analysis of Cross Section and Panel Data. MIT Press, Cambridge (2002) 